\title{
Pseudoaneurisma micótico por Mycobacterium tuberculosis
}

\author{
C. MARTÍNEZ-VÁZQUEZ, A. NODAR, M. CRESPO, M. SEIJAS, D. CID, A. LÓPEZ, \\ R. BOUZAS* \\ Unidad de Enfermedades Infecciosas. Servicio de Medicina Interna. *Servicio de \\ Radiodiagnóstico. Complejo Hospitalario Xeral-Cíes Vigo. Universidad de Santiago de \\ Compostela
}

\author{
MYCOTIC PSEUDOANEURYSM BY MYCOBACTERIUM \\ TUBERCULOSIS
}

\begin{abstract}
RESUMEN
La tuberculosis puede afectar a cualquier órgano de la economía, incluidas las arterias. Habitualmente esta afectación se produce por contigüidad de la arteria a un foco infeccioso adyacente, originándose fundamentalmente pseudoaneurismas.

Describimos un caso de pseudoaneurisma tuberculoso de arteria ilíaca en probable relación con una peritonitis tuberculosa, que debutó como fiebre de origen desconocido. El diagnóstico de pseudoaneurisma se fundamentó en $\mathrm{CT}$, confirmándose mediante estudio anatomopatológico y microbiológico obtenido en una primera intervención quirúrgica. A pesar del tratamiento tuberculostático, el paciente presentó rotura del pseudoaneurisma, lo que obligó a implantar un injerto. Creemos que no debe olvidarse la posibilidad de una afectación tuberculosa en un aneurisma micótico.
\end{abstract}

PALABRAS CLAVE: Aneurisma micótico. Tuberculosis. Pseudoaneurisma. Peritonitis tuberculosa.

\begin{abstract}
Mycobacterium tuberculosis can involve any organ of the body, including the arteries. Usually the mycobacteria infects the arterial wall spreading from a contiguous foci. We report a case of tuberculous pseu doaneurysm involving the iliac artery clinically manifested as fever of unknown origin. The diagnosis relied on abdominal CT-scanning. Tuberculous etiology was confirmed postoperatively by microbiologic and microscopic study. The antituberculous therapy was early started, but the patient died three months later as a consequence of a non-infectious abdominal aoric rupture. Clinicians should consider tuberculous etiology when the diagnosis of mycotic pseudoaneurysm is being enter tained.
\end{abstract}

KEY WORDS: Mycotic pseudoaneurysm. Tuberculosis. Pseudoa neurysm. Tuberculous peritonitis.

Martínez-Vázquez C, Nodar A, Crespo M, Seijas M, Cid D, López A, Bouzas R. Pseudoaneurisma micótico por Mycobacterium tuberculosis. An Med Interna (Madrid) 2001; 18: 594-596.

\section{INTRODUCCIÓN}

A pesar de lo poco correcto del término, denominamos aneurisma micótico a cualquier dilatación arterial local infectada, sea o no un hongo su agente causal (1). Al contrario de lo que ocurre en el verdadero aneurisma micótico, en el que estructuras de la pared arterial constituyen el saco aneurismático, en el pseudoaneurisma micótico no se identifican dichas estructuras, y sí el material inflamatorio contiguo a la pared, procedente en parte de fugas hemáticas provocadas por dicha infección (1). La infección puede asentar sobre una pared arterial sana o aterosclerótica, siendo la fuente de infección más frecuente intravascular, si bien existen casos en donde la infección arterial se produce por contigüidad.

La implicación de Mycobacterium tuberculosis en infecciones arteriales es excepcional, recogiéndose en una revisión clásica de la literatura del año 1965 aproximadamente 100 casos, de los cuales el $50 \%$ se comportaron como pseudoaneurismas (2).

La reciente oportunidad de diagnosticar y tratar a un paciente con pseudoaneurisma tuberculoso nos motiva a publicar este trabajo.

\section{CASO APORTADO}

Paciente de 87 años con antecedentes de prostatismo y fibrilación auricular crónica que ingresa en el servicio de Cirugía Vascular de nuestro centro procedente de otro hospital por cuadro febril de tres meses y medio de duración y sospecha clínica y radiológica de aneurisma micótico de arteria ilíaca izquierda. Durante este tiempo y hasta la realización del primer TAC abdominal, el paciente había recibido múltiples pautas antibióticas empíricas sin mejoría clínica alguna. Además de la fiebre, había presentado dolorimiento a nivel abdominal que se incrementaba a la palpación profunda en fosa ilíaca izquierda.

Trabajo aceptado: 20 de Enero de 2000

Correspondencia: César Martínez-Vázquez. Calle Gran Vía nº 3-2º 36204 Vigo. 
Los distintos análisis realizados hasta ese momento, incluidos varios hemocultivos habían resultado siempre normales o negativos.

Cuando vemos por primera vez al paciente está consciente, orientado, se aprecia palidez cutáneo-mucosa, un soplo sistólico III/VI audible en todos los focos, con irradiación a carótida, hipofonesis en base pulmonar izquierda y zona dolorosa y algo empastada en fosa ilíaca derecha. El resto de la exploración física por aparatos y sistemas fue normal.

En la analítica general realizada a su ingreso destaca una anemia de 9,5 g. de Hb, normocítica y normocrómica, 6.220 leucocitos/mmc. con fórmula normal, 292.000 plaquetas/mmc. VSG $105 \mathrm{~mm} / \mathrm{h}$. Albúmina 2,4 g/dl. Colesterol $104 \mathrm{mg} / \mathrm{dl}$. Triglicéridos $94 \mathrm{mg} / \mathrm{dl}$. Perfil de enzimas hepáticos y función renal normal. Gasometría arterial normal. Sedimento urinario normal. Hemocultivos realizados a su ingreso y tras ventana de 48 horas sin antibióticos negativos. Serologías para fiebre Q, Toxoplasma, Brucella, Salmonella, VEB, CMV y VIH negativas para enfermedad reciente. ANA negativos., TSH y T4 normales. Alfa fetoproteína y CEA normales. Coprocultivo y urocultivo negativos. Rx de tórax: discreto derrame pleural izquierdo. Líquido pleural: ph 7,54, 1,800 leucos/mmc. (predominio de mononucleares), proteinas totales 3,8 g/dl. Glucosa $119 \mathrm{mg} / \mathrm{dl}$. LDH $255 \mathrm{UI} / \mathrm{l}$. ADA 30,20 U/1 (hasta 45 U/1). Citología: negativa para malignidad. Cultivo y BAAR negativos. ECG: fibrilación auricular con buena respuesta ventricular. Ganmagrafía de Ventilación/Perfusión: de baja probabilidad para tromboembolismo pulmonar. Ecocardiografía: Velos aórticos con cambios degenerativos que no reducen la apertura valvular. Sin evidencia de lesiones sugestivas de endocarditis. Ganmagrafía corporal con Galio: captación anómala y patológica a nivel de zona pélvica derecha. TAC helicoidal abdominal: masa abdómino-pelvica en íntima relación con ilíaca común derecha de $6 / 2,5 \mathrm{cms}$. de diámetro, con captación heterogénea de contraste, multiseptada; enfermedad aterosclerótica difusa con arteriopatía obliterativa del cono de la aorta hacia ambas ilíacas; ulceración de la ilíaca comun derecha, profunda hacia esa masa y afectación mesentérica con patrón estrellado, y con engrosamiento del omento mayor (Fig. 1). Ante los datos radiológicos se decide realizar intervención quirúrgica, apreciándose tras la apertura de la cavidad abdominal unas asas intestinales pegadas íntimamente entre si, evidenciándose en el retroperitoneo una masa de coloración oscura, no pulsátil y de aspecto necrótico. Las distintas biopsias tomadas de dicha masa son informadas como infiltrado mononuclear inespecífico e intensa necrosis, y las biopsias de epiplon evidenciaron la existencia de inflamación granulomatosa, siendo la investigación para BAAR positiva, tanto anatomopatológicamente como en el laboratorio de Microbiología. Posteriormente se confirmó la presencia de Mycobacterium tuberculosis en las muestras procedentes de la masa y del epiplon. No se implantó en esta intervención ninguna prótesis vascular. Se inició tratamiento con cuatro fármacos (isoniacida+rifampicina+etambutol+pirazinamida), apreciándose una desaparición de la fiebre, así como una franca mejoría clínica del paciente. Diez días después del inicio del tratamiento, de manera brusca, el paciente presentó un dolor intenso a nivel infraumbilical derecho, evidenciándose a la palpación una masa globulosa y dolorosa de aproximadamente $10 \mathrm{cms}$. de diámetro. Se realizó una TAC helicoidal apreciándose una extravasación sanguínea a nivel de la masa parailíaca común derecha (Fig. 2). El paciente fue sometido a una nueva laparotomía con ligadura de la ilíaca común derecha e implantación de un by-pass termino-lateral bifemoral. Se continuó con el tratamiento tuberculostático, apreciándose una buena evolución clínica y radiológica en nuevo TAC de control realizado previamente al alta hospitalaria. Tres meses más tarde de forma súbita inicia de nuevo dolor en región infraumbilical derecha, confirmándose mediante TAC nuevo resangrado debido a rotura de la pared posterior de la Aorta de aspecto no inflamatorio, siendo necesaria una nueva intervención con implantación de by-pass aorto-ilíaco izquierdo y femoral derecho. Durante el postoperatorio inmediato, el paciente presentó inestabilidad hemodinámica y parada cardiorrespiratoria, sin responder a las medidas de reanimación habituales. No existe estudio necrópsico.



Fig. 1. TAC helicoidal. Corte axial sumado a reconstrucción tridimensional de la aorta, ulceración de la arteria ilíaca común derecha, en contacto con masa abdómino-pélvica, marcas vasculares engrosadas en relación con mesenterio estrellado.

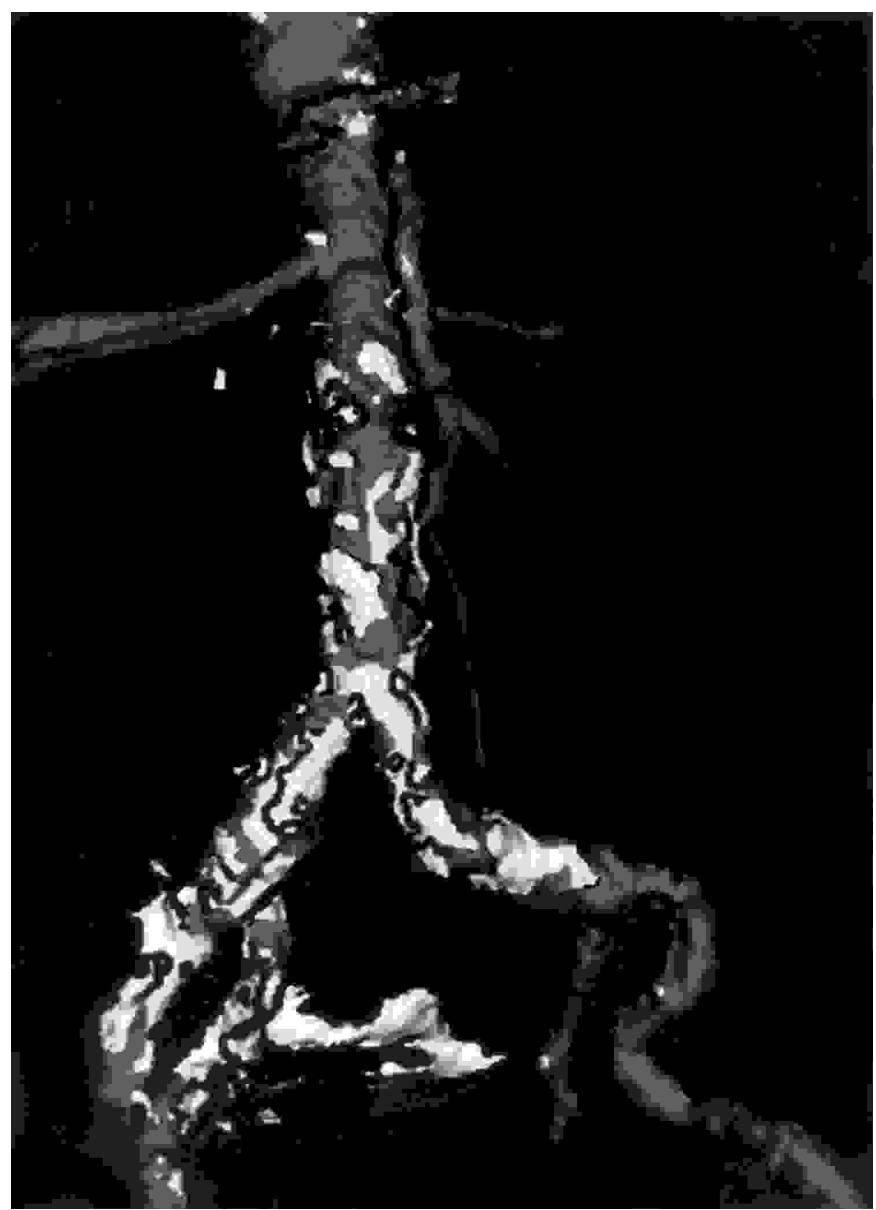

Fig. 2. TAC helicoidal. Reconstrucción tridimensional de la aorta abdominal y arterias ilíacas, ulceración profunda con formación de pseudoaneurisma y pared irregular al mismo nivel donde se había detectado la masa. 


\section{DISCUSIÓN}

Coincidiendo con la epidemia de infección por VIH durante las dos últimas décadas, se ha producido un nuevo brote de tuberculosis (3); sin embargo continua siendo poco frecuente la afectación arterial por Mycobacterium tuberculo sis, habiéndose descrito un solo caso de afectación arterial tuberculosa relacionado con la infección por VIH (4).

Los aneurismas micóticos tuberculosos suelen producirse por extensión directa de un foco tuberculoso adyacente, frecuentemente de una linfadenitis $(5,6)$, aunque también se ha descrito a partir de una afectación pericárdica, pleural, esofágica (7) o de una enfermedad de Pott (8). Aunque en nuestro caso no podemos asegurar que el foco primario sea la peritonitis tuberculosa, la ausencia de otro foco o de una tuberculosis miliar, así como la localización de la lesión arterial, sugiere fuertemente que este caso se deba a una afectación por extensión directa de una peritonitis tuberculosa, lo que convierte al caso en poco frecuente. Esta afectación por contigüidad explicaría la mayor frecuencia de pseudoaneurismas que de aneurismas verdaderos; el foco tuberculoso se extendería a través de la pared arterial, produciendo una pequeña perforación transmural, formando así un hematoma que constituiría la pared del pseudoaneurisma $(5,9)$. Los casos en los que no se identifica un foco contiguo se creen debidos a una diseminación hematógena de los bacilos, bien a través de los vasa vasorum o por implantación en la íntima, frecuentemente sobre una aorta enferma (9). La ausencia de casos de aneurismas tuberculosos en series de pacientes con tuberculosis miliar sugiere que esta diseminación hematógena sea el resultado y no la causa del aneurisma (6).

La clínica depende fundamentalmente del tamaño, de la localización, de la velocidad de crecimiento, que suele hacerse evidente en el plazo de una semana o incluso menos (5), y de la actividad de la infección tuberculosa (9). Los síntomas característicos son, al igual que en nuestro paciente, fiebre y dolor, aunque no siempre se encuentran presentes (6). Ante la aparición de un deterioro brusco en estos pacientes, se ha de sospechar la existencia de una rotura del aneurisma o pseudoaneurisma, que es la complicación más frecuente, y puede producirse hacia el pulmón, pleura, pericardio, espacio retroperitoneal, esófago, sigma o duodeno (10), dependiendo de la localización. Nuestro caso se comportó como una fiebre de origen desconocido en un principio, y a pesar de estar con tratamiento tuberculostático presentó sangrado del pseudoaneurisma que obligó a una segunda intervención. En cuanto al diagnóstico, son imprescindibles las técnicas de imagen, como la TAC o la arteriografía, además de la búsqueda de una tuberculosis a otro nivel. En nuestro caso la TAC aportó la sospecha de afectación peritoneal tuberculosa y el estudio helicoidal precisó la participación de la pared arterial en el proceso. El tratamiento ha de incluir necesariamente un abordaje quirúrgico acompañando al tratamiento médico. El seguimiento ha de ser a largo plazo, por la posibilidad de una reactivación del proceso tuberculoso o por el desarrollo de afectación vascular a otros niveles $(2,6)$. Dada la agresividad del proceso está indicada una escisión precoz junto con un tratamiento prolongado; este tratamiento puede mejorar la alta mortalidad de estos pacientes (11). Nuestro paciente falleció después de una tercera intervención, supuestamente por hemorragia de aneurisma aórtico abdominal no micótico, por lo que no podemos conocer cual hubiese sido la evolución del pseudoaneurisma tuberculoso.

La infección tuberculosa puede afectar a cualquier órgano de la economía, debiendo mantenerse la sospecha diagnóstica de afectación arterial en caso de que el cuadro clínico sea semejante al descrito con anterioridad.

\section{Bibliografía}

1. Scheld WM, Sande MA. Endocarditis and intravascular infections. En: Mandell, Douglas, Bennett. Principles and pactice of infectious diseases. Churchill Livingstone Inc, 1995; 740-82.

2. Silbergleit A, Arbulu A, Defever BA, Nedwiki EG. Tuberculous aortitis: surgical resection of ruptured abdominal false aneurysm. JAMA 1965; 193: 333-5.

3. Hopewell P. Tuberculous in persons with human inmunodeficiency virus. En: M. Sande, P. Volderbing. The medical management of AIDS. 5th ed. Philadelphia: WB Saunders, 1997; 311-25.

4. Gouny P, Valverde A, Vincent D, Fadel E. Human inmunodeficiency virus and infected aneurysm of the abdominal aorta:report of three cases. Ann Vasc Surg 1992; 6(3): 239-43.

5. Felson B, Akers PV, Hall G, Schreiber JT, Greene RE, Pedrosa CS Mycotic tuberculous aneurysm of the toracic aorta. JAMA, 1977; 237: 1104-8.

6. Estrera AS, Platt MR, Mills LJ, Nikaidoh H. Tuberculous aneurysm of

the descendenting thoracic aorta. Report of a case with fatal rupture Chest 1979; 75, 3: 386-9.

7. Catinella FP, Kittle CF. Tuberculous esophagitis with aortic aneurysm fistula. Ann Thorac Surg 1988; 45(1): 87-8.

8. McHenry MC, Rehm SJ, Krajewski LP, Duchesneau PM, Levin HS, Steinmuller DR. Vertebral osteomyelitis and aortic lesions: case report and review. Rev Infect Dis 1991; 13(6): 1184-94.

9. Efremidis SC, Lakshmanan S, Hsu JT. Tuberculous aortitis: a rare case of mycotic aneurism of the aorta. Am J Roentgenol 1976; 127: 859-61.

10. Goldbaum TS, Lindsay J Jr, Levy C, Silva CA. Tuberculous artitis presenting with an aortoduodenal fistula: a case report. Angiology 1986; 37 (7): 519-23.

11. Ikezawa T, Iwatsuka Y, Naiki K, Asano M, Ikeda S, Kimura A. Tuberculous pseudoaneurysm of the descending thoracic aorta: a case report and literature review of surgically treated cases. J Vasc Surg 1996; 24 (4): 693-7. 